\title{
Feasibility Study of Shell Powder as Sludge Conditioning Agent
}

\author{
Zhongwei Zhang ${ }^{1,2}$, Yubo Cui ${ }^{1,2, *}$, Junwen $\mathrm{Ma}^{1}$, Wanjun Zhang ${ }^{1,2}$, and Zhaobo Chen ${ }^{1,2}$ \\ ${ }^{1}$ Key Laboratory of Biotechnology and Bioresources Utilization, Ministry of Education, Dalian Minzu University, Dalian 116600, China \\ ${ }^{2}$ College of Environment and Resources, Dalian Minzu University,Dalian 116600 , China
}

\begin{abstract}
Sludge becomes a "world problem" with the increase of wastewater treatment amounts across the world. Sludge treatment is mainly for reduction, stabilization, harmlessness and recycling. By adding sludge conditioning agents, it can effectively reduce the moisture content and the specific resistance of sludge, and provide effective guarantees for subsequent sludge dewatering. The shells were used to reduce the moisture content and specific resistance of sludge herein. Through the analysis of experimental data, the results showed that the best dosage of shell powder solution was $6 \%$ of the dry weight of the sludge, and the best dosage of shell powder was $24 \%$ of the dry weight of the sludge. Under the optimal dosage, changing the $\mathrm{pH}$ value could also cause changes in the water content and the specific resistance of the sludge. The optimal $\mathrm{pH}$ values of the shell powder solution and shell powder for sludge conditioning were 8-9 and 7, respectively.
\end{abstract}

\section{Introduction}

With the acceleration of urban processes and the improvement of quality of life in cities around the world, the discharge of industrial wastewater and domestic sewage is increasing, and the output of sludge is rapidly increasing. Sludge is an inevitable product of sewage treatment plants and sewage treatment. When sludge that has not been properly disposed of enters the environment, it will directly cause secondary pollution to water bodies and the atmosphere, which not only reduces the effective treatment capacity of the sewage treatment system, but also poses a serious threat to the ecological environment and human activities. Sludge dewatering is a key part of sludge treatment and disposal, but no matter how it is treated, sludge dewatering is a necessary process. The water content of the sludge is above $95 \%$, and its water mainly exists in two forms of pore water and capillary water. The sludge is not only difficult to transport, but also not conducive to subsequent treatment and disposal. Therefore, a sludge conditioning agent is needed to convert the water in the sludge to free water or interstitial water, which is beneficial to the subsequent mechanical dehydration and improves the dehydration efficiency. At present, sludge conditioning technology is mainly divided into four categories: chemical conditioning, biological conditioning, physical conditioning and joint conditioning, of which chemical conditioning is currently the most widely used ${ }^{[1]}$.

The living standards of the vast water resources of the Chinese people are increasing day by day, resulting in the rapid development of shellfish breeding industry. China is a large shellfish farming country, and the output of marine shellfish farming accounts for about $80 \%$ of the total output of marine farming. Shells contain a lot of minerals, which have been used in new composite building materials, medicine, jewelry and other industries. It is known that when calcium carbonate is used as a chemical conditioning agent, it can effectively increase the porosity of sludge and promote the separation of sludge and water ${ }^{[2]}$. In the natural world, shell shells contain a large amount of calcium carbonate, which accounts for $95 \%$ of the whole shell, and the shell structure of the shell is special ${ }^{[3]}$, its prismatic layer contains a large number of $2 \sim 10$ gm pores, calcium carbonate It can also be used as a natural $\mathrm{pH}$ adjuster. $\mathrm{Ca}^{2+}$ in calcium carbonate greatly promotes the negatively charged colloidal particles, reduces the repulsive force between the particles, the shell structure is stable, and has a certain bridging effect ${ }^{[4]}$. In summary, the natural conditions of the shell provide an effective theoretical basis for the shell powder as a sludge conditioning agent. This article verifies the feasibility of using shell powder as a sludge conditioning agent and also provides a reference for subsequent experimental research.

\section{Materials and methods}

\subsection{Materials}

The sludge used in this experiment was taken from the first sewage treatment plant in Dalian Development Zone. The measured sludge moisture content was about $91.2 \%$, the specific resistance of the sludge was $5.5^{*} 10^{11} \mathrm{~m} \cdot \mathrm{kg}^{-1}$, and the dry sludge quality per $100 \mathrm{~mL}$ of sludge was $0.5476 \mathrm{~g}$; grind the shell with a grinder to make shell powder; hydrochloric acid; $\mathrm{NaOH}$ solution; experimental instrument: stopwatch; filter paper; oven;

\footnotetext{
* Corresponding author: cyb@dlnu.edu.cn
} 
Buchner funnel; thermometer; $\mathrm{pH}$ measuring instrument, coagulation stirrer.

\subsection{Methods}

All reagents were prepared at an ambient temperature of $25 \pm 2^{\circ} \mathrm{C}$ using distilled water. Unless otherwise stated, the experimental procedures were as follows: First configure a certain proportion of shell powder solution, then add the shell powder solution to the sludge to adjust, measure the specific resistance of the sludge under different dosages, so as to obtain the best dosage. Adjust the $\mathrm{pH}$ at the optimal dosage, and get the optimal $\mathrm{pH}$ after data analysis.; secondly, add shell powder directly to the sludge to adjust, the steps are the same as above. All experiments were conducted in triplicate, and averaged values were used for data analysis. Finally through the data integration analysis, the shell powder is used as a sludge conditioning agent to reduce pollution The effect of mud specific resistance.

\subsection{Sludge parameter measurement}

\subsubsection{Moisture content}

Take $100 \mathrm{~mL}$ of sludge, place the weighed filter paper on the Buchner funnel, moisten the filter paper with water, and press it against the bottom of the funnel. Start the vacuum pump, adjust the vacuum pressure, stabilize about $0.035 \mathrm{MPa}$, and turn off the vacuum pump. Pour $100 \mathrm{~mL}$ of sludge into the Buchner funnel, start the vacuum pump, adjust the vacuum pressure to the experimental pressure, and filter until the vacuum is broken. Close the valve and remove the filter cake to weigh in the balance. The weighed filter cake is weighed after being dried in an oven at $105^{\circ} \mathrm{C}$, and the moisture content of the sludge is obtained by calculation.

\subsubsection{The specific resistance of sludge}

Take $100 \mathrm{~mL}$ of sludge in the Buchner funnel, start the vacuum pump, adjust the pressure to the experimental pressure; after reaching this pressure, start the stopwatch, and note the filtrate in the metering tube when it is started, and record the corresponding amount of filtrate in the metering tube at regular intervals, Filter until the vacuum is broken (if the vacuum is not broken for a long time, the filtration can be stopped after 20 minutes). Close the valve, remove the filter cake and put it into the balance for weighing. The weighed filter cake is dried and weighed in an oven at 105, and the specific resistance of sludge is calculated.

Table 1. Sludge experiment parameters.

\begin{tabular}{|c|c|c|c|}
\hline $\begin{array}{c}\text { Vacuum } \\
\text { pressure } \\
(\mathrm{MPa})\end{array}$ & $\begin{array}{c}\text { Filter area } \\
\left(\mathrm{cm}^{2}\right)\end{array}$ & $\begin{array}{c}\mathrm{SRF} \\
(\mathrm{m} / \mathrm{kg})\end{array}$ & $\begin{array}{c}\text { Moisture } \\
\text { content } \\
(\%)\end{array}$ \\
\hline 0.035 & 63.56 & $5.5^{*} 10^{11}$ & 91.20 \\
\hline
\end{tabular}

\section{Results and discussion}

\subsection{Effect of shell powder solution on water content and sludge specific resistance}

As shown in Figure 1, when the shell powder is not added, the mud cake moisture content is $91.2 \%$; as the shell powder solution dosage increases, the mud cake moisture content shows a downward trend, When the dosage of the shell powder solution is $12 \%$, the moisture content of the mud cake is $87.7 \%$, and the moisture content is below the original mud. After adding the Baker powder solution, the specific resistance of the sludge is less than that of the original mud. When the dosage of the shell powder solution is $6 \%$, the specific resistance of the sludge is at least $3.19 * 10^{11} \mathrm{~m} \cdot \mathrm{kg}^{-1}$, at $9 \%$ The specific resistance of the sludge is higher than that of $6 \%$ and $12 \%$, but the specific resistance of the sludge at $12 \%$ is $3.20 * 10^{11} \mathrm{~m} \cdot \mathrm{kg}^{-1}$, which is little different from $6 \%$. As the amount of shell powder solution increased, the specific resistance of sludge gradually decreased. When the dosage of shell powder solution is $6 \%$, it has a significant effect of reducing, which shows that the shell powder solution has a certain improvement effect on the sludge dewatering performance. Therefore, the optimal dosage of shell powder solution is set to $6 \%$ of the mass of dry sludge.

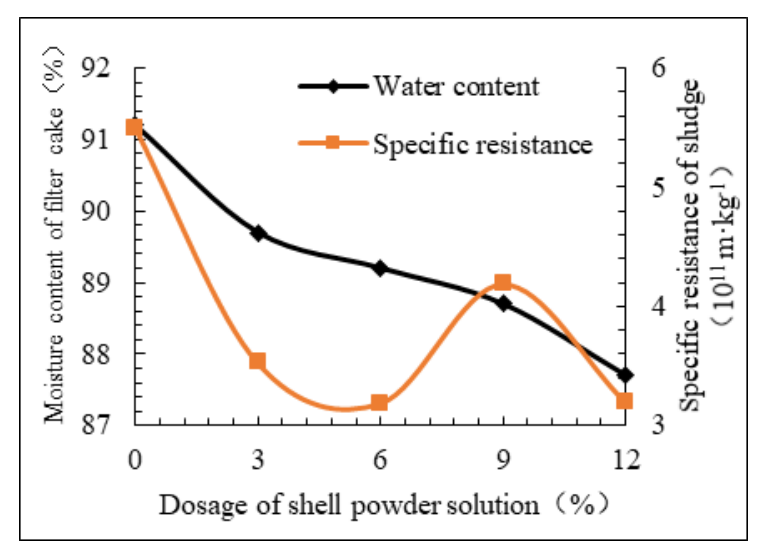

Fig. 1. Effect of shell powder solution on water content and sludge specific resistance.

\subsection{Influence of adjusting $\mathrm{pH}$ on water content and sludge specific resistance}

The best dosage of shell powder solution is $6 \%$. From Figure 2, when the amount of shell powder solution is unchanged, as the $\mathrm{pH}$ value of the sludge increases, the moisture content of the mud cake first decreases and then increases, reaching the lowest when the $\mathrm{pH}$ is 8 , and the moisture content of the mud cake is $84.6 \%$.

As the $\mathrm{pH}$ value increases, the specific resistance of sludge first decreases, and then continues to increase after reaching the lowest point. When the $\mathrm{pH}$ is 9 , the specific resistance of sludge reaches a minimum of 2.92 $* 10^{11} \mathrm{~m} \cdot \mathrm{kg}^{-1}$. When the dosage of shell powder solution is $6 \%$, when the $\mathrm{pH}$ value of sludge is between $8-9$, the effect of shell powder solution on reducing sludge specific resistance is the best. 


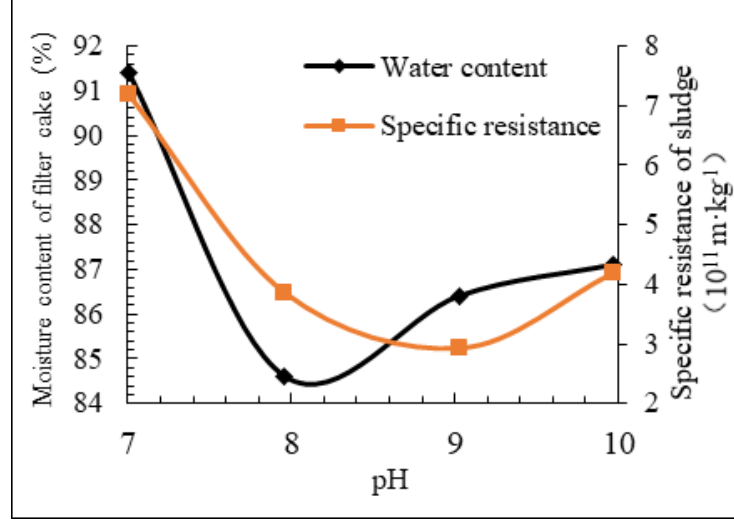

Fig. 2. Effect of $\mathrm{pH}$ on water content and sludge specific resistance.

\subsection{Influence of shell powder on water content and specific resistance of sludge}

Because the main component of shell powder is calcium carbonate, and calcium carbonate is difficult to dissolve in water, so this experiment needs to increase the content of shell powder. As shown in Figure 3, as the amount of shell powder added increases, the water content of the filter cake first decreases and then increases and then decreases. When the dosage is $24 \%$ of the dry sludge mass, the water content of the filter cake reaches a minimum of $78.20 \%$. That is to say, the moisture content of the sludge cake prepared by adding shell powder is below the original mud. It can be seen from Figure 3 that the addition of shell powder has a certain effect on the reduction of the specific resistance of sludge. When the amount of shell powder is $24 \%$, the specific resistance of sludge is the lowest, which is $3.48 * 10^{11} \mathrm{~m} \cdot \mathrm{kg}^{-1}$ Since the specific resistance of sludge has a significant effect of reducing the specific resistance when the shell powder dosage is $24 \%$, it is determined that the optimal amount of shell powder is $24 \%$ of the dry sludge mass.

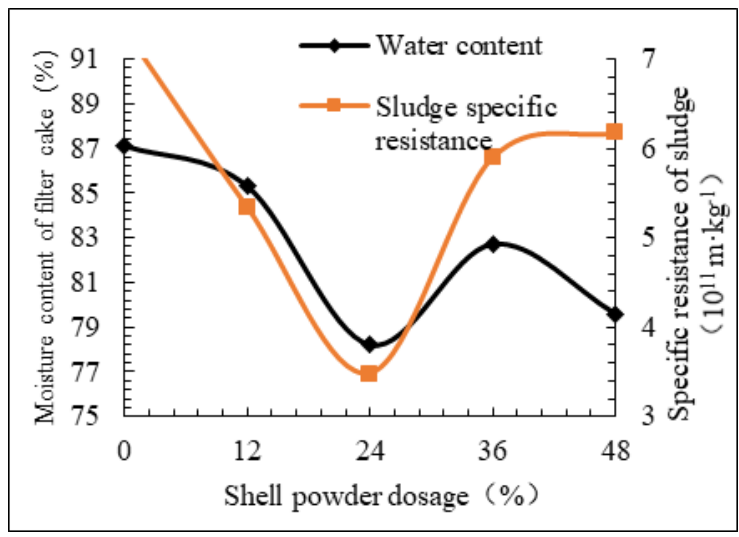

Fig. 3. Effect of shell powder on moisture content and sludge specific resistance.

\subsection{The effect of $\mathrm{pH}$ on water content and sludge specific resistance}

The best shell powder dosage is $24 \%$. As shown in Figure 4 , as the $\mathrm{pH}$ increases, the moisture content of the mud cake first decreases, decreases to a certain level, then increases and then decreases. At $\mathrm{pH}=7$, the moisture content of the filter cake reaches the lowest. At the same time, as the $\mathrm{pH}$ value increases, the specific resistance of sludge first decreases and then increases. The specific resistance of sludge reaches the lowest value at $\mathrm{pH}=7$. Therefore, when the shell powder dosage is $24 \%$ and $\mathrm{pH}=7$, Shell powder is the best sludge conditioning agent to reduce sludge specific resistance.

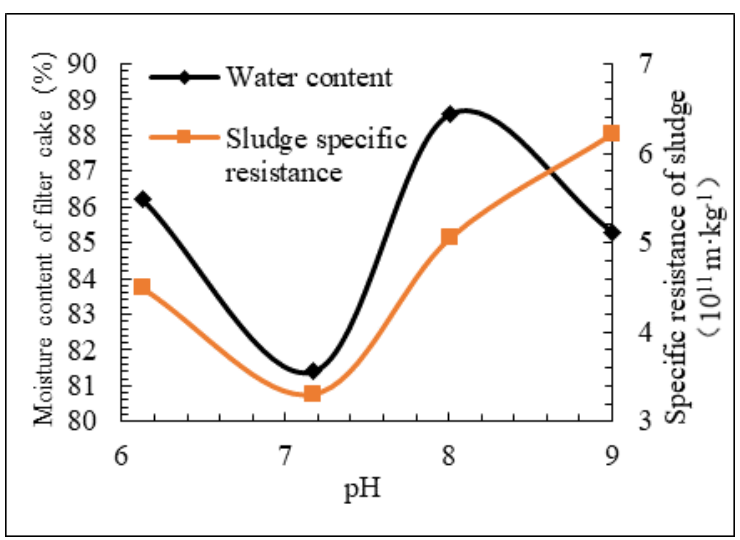

Fig. 4. Effect of $\mathrm{pH}$ on water content and sludge specific resistance.

\section{Conclusions and expectation}

This paper mainly studies the effect of grinding waste shells into powder as a coagulant to improve the dewatering performance of sludge, the impact on the specific resistance of sludge and the moisture content of filter cake. Through the above experiments and data processing analysis, the following conclusions can be drawn:

The addition of shell powder solution and shell powder can reduce the specific resistance of sludge and increase the solid content of mud cake; but the effect of adding shell powder is not as good as that of adding shell solution;

In the case of adding the same amount of sludge conditioning agent, changing the $\mathrm{pH}$ value will affect the specific resistance of the sludge. For adding shell powder solution, the optimal $\mathrm{pH}$ is between 8 and 9, while for shell powder, the optimal $\mathrm{pH}$ is around 7.

The above conclusions indicate that the use of shell powder in coagulant has considerable research value. The perfect combination of shell structure and physical properties is a masterpiece of nature, and its comprehensive utilization should be studied in depth to provide beneficial help for subsequent research.

\section{Acknowledgements}

The research was financed by the Science and Technology Innovation Foundation of Dalian, China (2018J12SN080) and Natural Science Foundation (National Innovation Joint Fund) of Liaoning, China. 


\section{References}

1. D.D. Chen, Y.H. Dou, P. Lu, et al. Chemical Industry and Engineering Progress, Research Progress of Deep Sludge Dewatering Technology, 38, 4722-4746 (2019)

2. X. Ma, H.H. Fan, M.Q. Lv, et al. Environ. Eng. Study on the Effect of Conditioner on the
Microstructure and Dewatering Performance of Sludge, 36, 113-116 (2018)

3. T. Cui. Hebei Fisheries. Research status of shell structure and application, 07, 47-50 (2019)

4. T. Cui. Hebei Fisheries. Application methods of shells and preparation of related materials, 41, 353358 (2019) 